\title{
Series Resistance in Vertical MOSFETs with Reduced Drain/Source Overlap Capacitance
}

\author{
L. Tan ${ }^{1}$, S. Hall ${ }^{1}$, O. Buiu ${ }^{1}$, M.M.A. Hakim ${ }^{2}$, T. Uchino ${ }^{2}$, P. Ashburn ${ }^{2}$, W. Redman-White ${ }^{2}$ \\ ${ }^{1}$ University of Liverpool, Brownlow Hill, Liverpool, L69 3GJ, UK \\ ${ }^{2}$ University of Southampton, Highfield, Southampton, SO17 1BJ, UK
}

\begin{abstract}
In this work we investigate the series resistances in vertical MOSFETs incorporating the fillet local oxidation (FILOX) structure that serves to reduce the gate to drain/source overlap capacitances. The series resistances are modeled analytically and the important influencing factors, namely gate bias dependence and the asymmetric nature of the device, are identified. We extract by simulation, $\mathbf{R}_{d}$ and $\mathbf{R}_{\mathrm{s}}$ from devices with different FILOX thicknesses, employing an impedance method often used in RF characterisation. We identify the trade-off whereby thickening the FILOX first causes an increase of the cut-off frequency $f_{T}$, until the on-current $I_{\text {on }}$ becomes limited by increasing series resistances and $f_{T}$ therefore reduces. The results indicate a thickness of $40 \mathrm{~nm}$ FILOX for maximum $f_{T}$. We also investigate the influence of process conditions on low series resistances, namely time of rapid thermal annealing RTA and angle of implantation.
\end{abstract}

\section{Introduction}

In our previous research into the feasibility of submicron Vertical MOSFETs (VMOST) for RF applications, a number of innovations have been proposed and their potential demonstrated in addressing device issues such as the overlap parasitic capacitance [1], short-channel effect [2] and parasitic bipolar effects [3]. As RF MOSFET scaling enters the deep micron regime, more attention is required on the effects of the drain and source series resistances on the overall device performance. It is already known that $R_{s}$ and $R_{d}$ exhibit gate bias dependence in MOSFETs [4]. It is also known that the device electrical performance and reliability, including the key performance indicators saturated drain current $\mathrm{I}_{\mathrm{on}}$, transconductance, noise figure, cut-off frequency and degradation due to hot carriers, depend more on source resistance $R_{s}$ than the drain resistance $R_{d}$ [5]. Therefore, in this work it is of interest to investigate series resistances in each junction of previously proposed vMOST concept with a fillet local oxidation (FILOX) structure [1]. Due to the asymmetrical characteristics of drain (top) and source (bottom) junction regions and the unique FILOX structure, it is important to obtain physical insight by first analytically modeling the gate bias dependence and asymmetric characteristics of the series resistance in relation to key metrics for further performance optimisation. The series resistances are extracted from a device generated from process simulation, using a small signal impedance method based on a practical RF measurement methodology. Next, series resistances for devices with different FILOX thicknesses are studied and an optimal value is identified based on a maximized gain-bandwidth product, $\mathrm{f}_{\mathrm{T}}$. Finally, some other key process parameters are identified in order to maintain low series resistances.

\section{Simulation setup}

2D computer simulations were carried out using the process simulator Athena Silvaco in accordance with the fabrication process as described in earlier work [1]. A sample device structure is shown in Fig.1 where FILOX is grown between the gate and the drain/source to reduce the overlap capacitances. Throughout this 'virtual experiment', the device channel length has been maintained at $100 \mathrm{~nm}$ with a gate oxide thickness of $4 \mathrm{~nm}$ and a body doping level of $1 \times 10^{18} \mathrm{~cm}^{-3}$. The FILOX thickness is varied from $20 \mathrm{~nm}$ to $60 \mathrm{~nm}$ while the RTA time and donor implant angle also vary in different experiments. The device simulations were conducted using the Silvaco Atlas simulation platform.

\section{Analytical model}

The series resistances in vMOST-FILOX can be modeled by the equivalent circuit shown in Fig.1a. The resistance components of concern occur along the current conduction path from the source to drain. In each junction, the series resistance is formed of three components: overlap region resistance $R_{o v}$, sheet resistance $R_{s h}$ and contact resistance $R_{c o}$. The scope of our work focuses on the overlap region resistance which constitutes a significant component of the total series resistance and also determines the asymmetric and gate bias dependencies. The overlap region resistance at the source junction is first analyzed from the schematic as shown in Fig.1b, where $R_{a c c}$ represents the accumulation layer resistance and $R_{\text {sp }}$ represents the spreading resistance.

The derivation of accumulation resistances follows a surface potential based approach for modeling the 

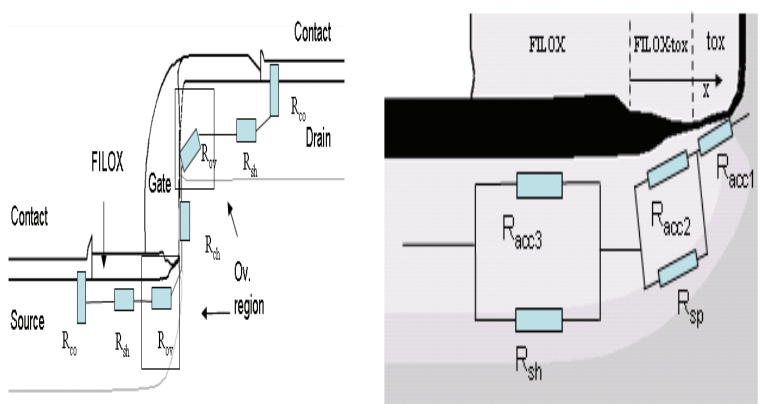

Fig1. a) A vMOST-FILOX structure ( $\mathrm{L}=100 \mathrm{~nm})$ with an equivalent circuit of the series resistance; $b$ ) Series

resistance in the overlap region of the source junction.

gate bias dependence of carrier charge density. This is unlike the work described in [6] where the surface potential in junctions was neglected and thus derivation of the gate bias dependence of accumulation layer charges was oversimplified. $R_{\text {accl }}$ under the uniform gate oxide is evaluated by integration of the local resistivity along the lateral junction depletion region. It can be expressed as below.

$$
R_{a c c 1}\left(\phi_{s}\right)=\int_{l_{\text {ext }}-W_{\text {dep }}}^{l_{\text {ext }}} \frac{d x}{w \cdot u_{e f f}\left(\phi_{s}\right) \cdot Q_{\text {sov }}\left(\phi_{s}, x\right)}
$$

Where $\phi_{\boldsymbol{S}}$ is the surface potential; $1_{\mathrm{ext}}$ is the total roll-off distance for a Gaussian doping profile that decreases from a level at two third of the maximum $\mathrm{N}_{\mathrm{dmax}}$ to $\mathrm{N}_{\text {sub }} ; \mathrm{W}_{\text {dep }}$ is the lateral depletion region width obtained from the Poisson equation and Gauss Law from the maximum electrical field, $\mathrm{E}_{\max }$, which is in turn, is obtained from a numerical simulation; the effective mobility in the accumulation layer is modeled by a universal accumulation layer carrier mobility model [7].

The spreading resistance $R_{s p}$ is evaluated by integrating the local resistivity over a region where the carriers spread into the junction bulk as the surface conduction reduces. The spreading angle is determined by the junction geometry and inversion layer thickness [8]. The results in Fig.2 show that $R_{s p}$ is much less compared to $\mathrm{R}_{\mathrm{acc} 2}$ which therefore dominates the FILOX- $t_{\text {ox }}$ region. In the FILOX region, the sheet resistance dominates. At the contact, the majority of the carriers flow into the metal at its edge rather than through the entire contact area [9]. Fig.2 also shows that the total series resistance is dominated by $\mathrm{R}_{\mathrm{accl}}$ and consequently shows the same gate bias dependence. This means that the device exhibits a smaller series resistance at high gate bias. *

In order to significantly reduce $R_{a c c l}$, we identify three key process parameters namely average junction

\footnotetext{
* The detailed analytical model will be described in the presentation
}

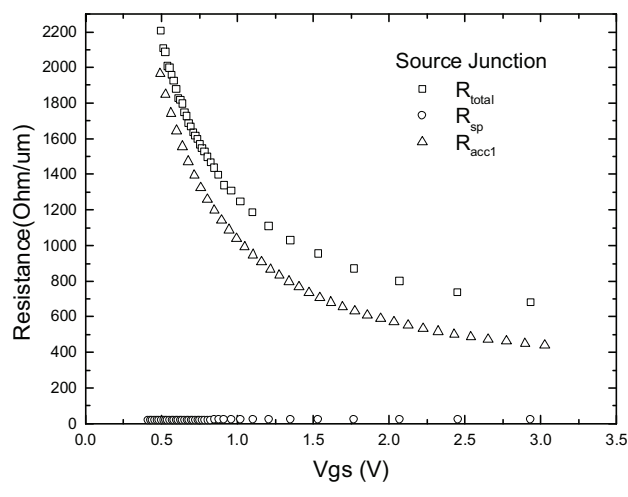

Fig.2 $R_{\text {accl }}, R_{\text {sp }}, R_{\text {total }}$ Vs. $V_{g s}$ of source junction

doping density, FILOX thickness encroachment and junction abruptness. Significantly, these parameters are different at the top and bottom of the vertical pillar as are the respective influences of the gate bias dependence and other asymmetric effects. FILOX tends to encroach more towards the junction boundary position at the pillar bottom for this case. The doping profile at the junction border also differs for the drain and the source due to differing dopant diffusion for vertical and lateral directions. Additionally, the distance between the drain contact edge and the vertical pillar surface also influences the drain resistance, $R_{d}$ by affecting the current spreading position that in turn influences the $R_{a c c 1}$ and $R_{s p}$.

\section{Series Resistance Extraction}

Many methods of extracting the combined series resistance $\left(\mathrm{R}_{\mathrm{d}}=\mathrm{R}_{\mathrm{s}}\right)$ from DC measurements have been reported and summarized in [10]. In these methods, an array of differing channel lengths, $\mathrm{L}$ are required and significant errors can be introduced for small values of L. A few DC methods [11, 12] have extracted $R_{d} / R_{s}$ separately taking account of its gate bias dependence using a single transistor, which is suitable for the case of vertical MOSTs with a small range of Ls. However, these methods require accurate determination of $\mathrm{L}_{\text {eff }}$ and complex calculations. In order to avoid all the aforementioned disadvantages, in this work we extract the series resistances from impedances of a MOSFET two port system [13]. During the extraction, the device is biased in the strong inversion region with $\mathrm{V}_{\mathrm{ds}}=0 \mathrm{~V}$ where the transadmittances and intrinsic gate-substrate capacitance, $\mathrm{C}_{\mathrm{gbi}}$ can be neglected. Additionally, the series resistances are extracted in the small signal test regime over a frequency range from $0.1 \mathrm{GHz}$ to $3 \mathrm{GHz}$ such that the impedances of junction-bulk capacitances can be neglected. The suitability of this frequency range for extraction is shown by the plot of resistances versus frequency in Fig.3. The extracted results of $R_{s}$ against $V_{g s}$ are illustrated in Fig.4 where the values are compared to the analytical model. The device fabrication process included a $0^{\circ}$ tilt source/drain arsenic implant and a 40s RTA at 


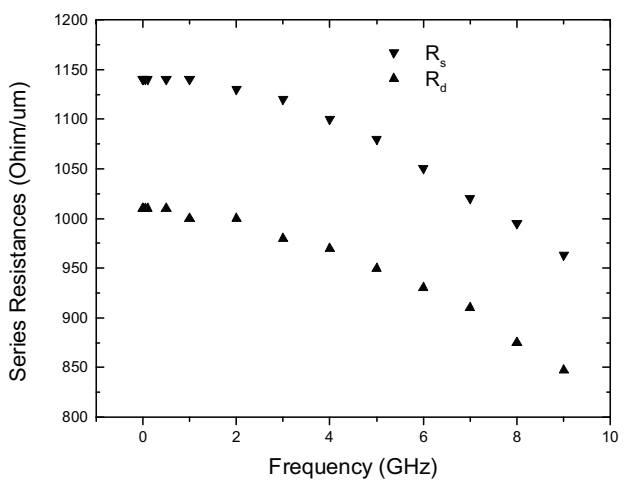

Fig. $3 R_{d} \& R_{s}$ vs. frequency, FILOX $=40 \mathrm{~nm}, 0^{\circ} \mathrm{S} / \mathrm{D}$ implant, 40s RTA, $\mathrm{V}_{\mathrm{gs}}=1 \mathrm{~V}, \mathrm{~V}_{\mathrm{ds}}=0 \mathrm{~V}$

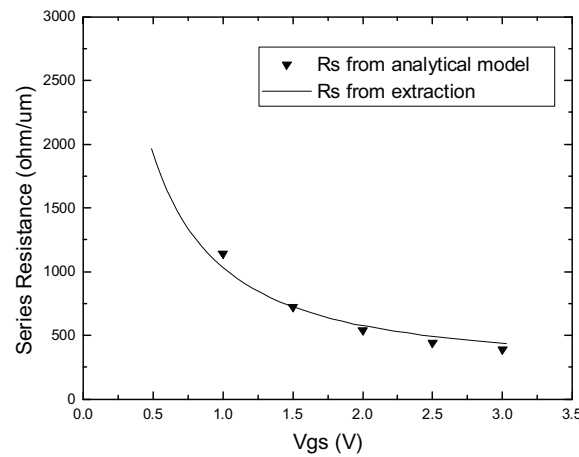

Fig. $4 \mathrm{R}_{\mathrm{s}}$ vs. $\mathrm{V}_{\mathrm{gs}}$, FILOX=40nm, $0^{\circ} \mathrm{S} / \mathrm{D}$ implant, 40s RTA, $\mathrm{V}_{\mathrm{ds}}=0 \mathrm{~V}$

$1100^{\circ} \mathrm{C}$.

To clarify the influence of FILOX thickness on the series resistance, we carried out extraction of $R_{d}$ and $\mathrm{R}_{\mathrm{s}}$ from simulation for the devices with FILOX thicknesses from $20 \mathrm{~nm}$ to $60 \mathrm{~nm}$. The results are illustrated and compared in Fig.5 which shows that series resistances experience a dramatic increase when FILOX thickness increases from $50 \mathrm{~nm}$ to $60 \mathrm{~nm}$. For a $60 \mathrm{~nm}$ FILOX, the analytical model indicates that the dramatic increase of $R_{s}$ is due to reduction of the junction doping level by thicker FILOX and its encroachment that has thickened the oxide above $\mathrm{R}_{\mathrm{accl}}$. Simulation has confirmed that the thickness of this oxide varies between $6 \mathrm{~nm}$ to $20 \mathrm{~nm}$ along the for the bottom junction. For the top junction with thicker FILOX, the resistance is increased due to junction doping level reduction only. Relatively less increase of $R_{d}$ compared to $R_{s}$ is because of much reduced FILOX encroachment into the oxide above $R_{\text {acc }}$.

The devices with thinner FILOX show much less variation in series resistances. The variation is mainly caused by varying junction doping level and junction abruptness for devices with different FILOX thicknesses. The small differences also indicate that for these devices, less asymmetry between $R_{s}$ and $R_{d}$ is due to significantly less FILOX encroachment into the sidewall near the bottom and top junction boundary, resulting in less variation of $\mathrm{R}_{\mathrm{acc}}$. Reducing the distance between the top contact edge and the sidewall by $50 \mathrm{~nm}$ in the top junction, causes

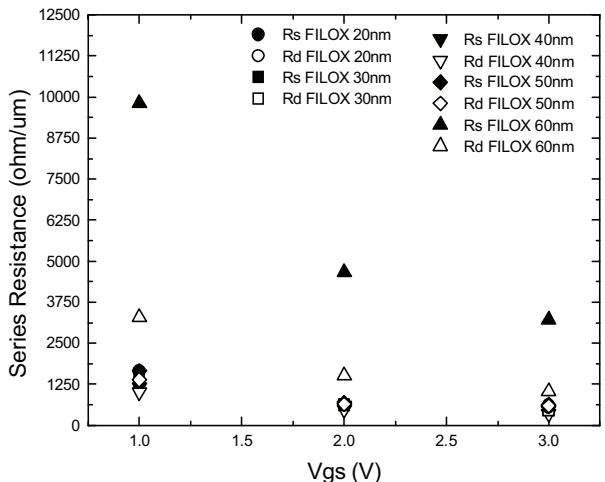

Fig. $5 \mathrm{R}_{\mathrm{d}}, \mathrm{R}_{\mathrm{s}}$ vs. $\mathrm{V}_{\mathrm{gs}}, 0^{\circ} \mathrm{S} / \mathrm{D}$ implant, 40s RTA, $\mathrm{V}_{\mathrm{ds}}=0 \mathrm{~V}$

an $11 \%$ increase in $R_{d}$.

The $\mathrm{f}_{\mathrm{T}}$ and $\mathrm{I}_{\mathrm{on}}$ for all the devices at $\mathrm{V}_{\mathrm{ds}}=\mathrm{V}_{\mathrm{gs}}=1.0 \mathrm{~V}$ are shown in Fig.6. It can be seen that $\mathrm{f}_{\mathrm{T}}$ increases with increase of FILOX thickness up to $40 \mathrm{~nm}$ and $\mathrm{f}_{\mathrm{T}}$ starts to fall thereafter. The increase of $\mathrm{f}_{\mathrm{T}}$ is due to the effect of decreasing overlap capacitance; the extracted total overlap capacitance values are listed in table.1 where a reduction with FILOX thickness can be seen. The decrease of $\mathrm{f}_{\mathrm{T}}$ beyond 40nm of FILOX is a result of the dominance of series resistance which limits $\mathrm{I}_{\mathrm{on}}$ and hence the transonductance, $\mathrm{g}_{\mathrm{m}}$. Therefore an optimal FILOX thickness of $40 \mathrm{~nm}$ is proposed for vMOSTs for a maximized $\mathrm{f}_{\mathrm{T}}$ performance.

A comparison among the series resistances from varied tilt angle of source/drain implantation and RTA process conditions is made and shown in Fig. 6 where the RTA temperature remains at $1100^{\circ} \mathrm{C}$ for all the cases. The results for $\mathrm{V}_{\mathrm{gs}}=2 \mathrm{~V}$ are listed in table 2. We observe that for a 40nm FILOX either 40 second RTA or 15 degree implantation is a necessary process step to extend the junction around the bottom corner and further into the pillar to avoid compromise of series resistance by FILOX encroachment. This is further assisted by using higher implantation energy that additionally increases the overall junction doping

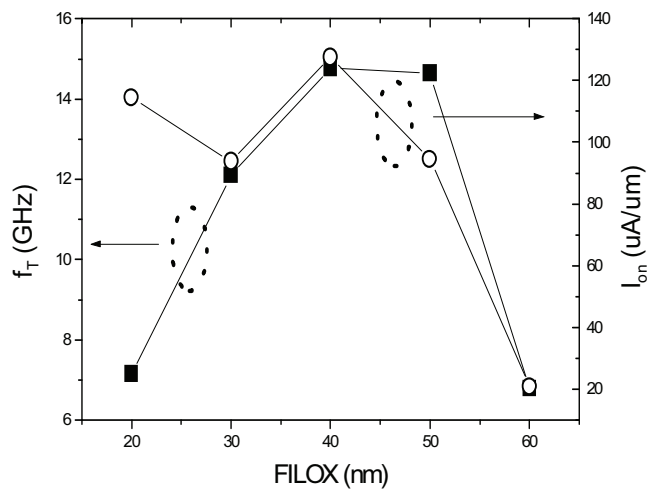

Fig.6 $\mathrm{I}_{\mathrm{on}}, \mathrm{f}_{\mathrm{T}}$ vs. FILOX thickness; $\mathrm{V}_{\mathrm{ds}}=\mathrm{V}_{\mathrm{gs}}=1.0 \mathrm{~V}$

Table.1 Overlap Capacitance vs. FILOX thickness

\begin{tabular}{|c|c|c|c|c|c|}
\hline $\mathrm{t}_{\text {FILOX }}(\mathrm{nm})$ & 20 & 30 & 40 & 50 & 60 \\
\hline $\mathrm{C}_{\text {overlap }}\left(\mathrm{fF} / \mathrm{um}^{2}\right)$ & 3.96 & 3.76 & 3.15 & 2.94 & 1.88 \\
\hline
\end{tabular}




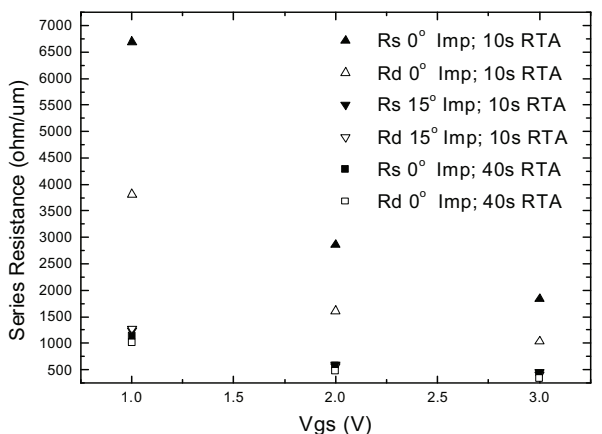

Fig. $6 R_{d}, R_{s}$ vs. $V_{g s}$ for cases of a) 0 degree $s / d$ implant, RTA=10s; b) 15 degree s/d implant, RTA=10s; c) 0 degree $\mathrm{s} / \mathrm{d}$ implant, RTA $=40 \mathrm{~s}$; all with FILOX $=40 \mathrm{~nm}, \mathrm{~V}_{\mathrm{ds}}=0 \mathrm{~V}$

Table. $2 \mathrm{R}_{\mathrm{d}}, \mathrm{R}_{\mathrm{s}}$ vs tilt angle, RTA time with $\mathrm{V}_{\mathrm{gs}}=2 \mathrm{~V}$

\begin{tabular}{|c|c|c|c|}
\hline Tilt Angle $\left({ }^{\circ}\right)$ & RTA time $(\mathrm{s})$ & $\mathrm{R}_{\mathrm{s}}(\Omega / \mu \mathrm{m})$ & $\mathrm{R}_{\mathrm{d}}(\Omega / \mu \mathrm{m})$ \\
\hline 0 & 10 & 2860 & 1610 \\
\hline 15 & 10 & 589 & 562 \\
\hline 0 & 40 & 581 & 479 \\
\hline
\end{tabular}

level. However, this is not true when the energy becomes too high that the peak of doping moves away from the surface of the bottom junction. Consequently $\mathrm{R}_{\mathrm{s}}$ deteriorates due to a reduced surface doping and less junction extension. In this case the short channel effect also becomes more severe due to a shortened channel length.

\section{Conclusions}

In this paper, an analytical and simulation study of series resistance components in vMOST-FILOX was presented. The key parameters that influence the $R_{d}$ and $R_{s}$ gate bias dependence and asymmetric device aspects were indentified. An impedance-RF method was used to extract $R_{d}$ and $R_{s}$ from devices with different FILOX thicknesses. The analytical model allowed insight into the dominant resistance components whereby apart from lowered junction doping level, the FILOX encroachment additionally increases the $R_{s}$. An optimal $f_{T}$ was seen for FILOX thickness of $40 \mathrm{~nm}$ which represented a trade-off between the dominance of series- $R$ limited $I_{o n}$ and the overlap capacitance. Finally the work also suggested that by using longer RTA time and angled tilt arsenic implantation for source/drain, the junction can be extended further away from the FILOX encroachment and the series resistance is efficiently reduced.

\section{References}

[1] V. D. Kunz, T. Uchino, C. H. Groot, P. Ashburn, D. C. Donaghy, E. Gili, S. Hall, Y. Wang, P. L. F. Hemment, "Reduction of parasitic capacitance in vertical MOSFET's by fillet local oxidation (FILOX)", IEEE Trans. on Electron Devices, vol. 50, pp. 1480-1487, June 2003.
[2] D. C. Donaghy, S. Hall, C. H. Groot, V. D. Kunz, P. Ashburn, "Design of a $50 \mathrm{~nm}$ vertical MOSFET incorporating a dielectric pocket", IEEE Trans. on Electron Devices, vol.ED-51, pp. 158-161, Jan. 2004

[3] V. D. Kunz, C. H. Groot, P. Ashburn, S. Hall, "Gain Control in SiGe HBTs by the Introduction of Germanium into Polysilicon Emitters", IEEE Trans. on Electron Devices, vol.ED-50, pp. 1481-1486, June 2003.

[4] G.J. Hu, C. Chang, Y Chia, "Gate-Voltage-Dependent Effective Channel Length and Series Resistance of LDD MOSFET's", IEEE Trans. on Electron Devices, vol. ED-34, p. $2469-2475,1987$

[5] J.F. Chen, J. Tao, P. Fang, C. Hu, "Performance and reliability comparison between asymmetric and symmetric LDD devices and logic gates", IEEE J Solid-State Circuits; vol. 34(3), pp.367-71 1999

[6] E.Gondro, F.Schuler, P.Klein, "A Physics Based Resistance Model of the Overlap Regions in LDD-MOSFETs", SISPAD, pp.267-270, 1998

[7] S. Mudanai, G..L. Chindalore, W.-K. Shih, H. Wang, Q. Ouyang, Al..F. Tasch, Jr., C. M. Maziar, S.K. Banerjee, "Models for Electron and Hole Mobilities in MOS Accumulation Layers", IEEE Trans. on Electron Devices, Vol.46, No.8, pp.1749-1759 1999

[8] S.D. Kim, C.M. Park, Jason C.S. Woo, "Advanced Model and Analysis of Series Resistance for CMOS Scaling Into Nanometer Regime - Part I: Theoretical Derivation", IEEE Trans. on Electron Devices, Vol. 49, No.3 pp.457-466, March 2002.

[9] H. Murrmann and Dietrich Widmann, "Current Crowding on Metal Contacts to Planar Devices", IEEE Trans. on Electron Devices, Vol.ED-16, No.12, December, 1969.

[10] S. Biesemans, M. Hendriks, S. Kubicek, and K.D. Meyer, "Practical Accuracy Analysis of Some Existing Effective Channel Length and Series Resistance Extraction Methods for MOSFET's", IEEE Trans. on Electron Devices, vol. 45, No.6, pp.1310-1316, June 1998

[11] C. Lou, W. Chim, D. Chan, Y. Pan, "A Novel Single-Device DC Method for Extraction of the Effective Mobility and Source-Drain Resistances of Fresh and Hot-Carrier Degraded Drain-Engineered MOSFET's", IEEE Trans. on Electron Devices, pp 1317-1323, vol. 45, No. 6, June 1998

[12] D.M. Kim, H.C. Kim, H.T. Kim, "Modeling and extraction of gate bias-dependent parasitic source and drain resistances in MOSFETs", Elsever, Solid State Electronics, vol. 47, pp 1707-1712, 2003

[13] E. Torres-Rios, R. Torres-Torres, G. Valdovinos-Fierro, E.A. Gutiérrez-D., "A Method to Determine the Gate Bias-Dependent and Gate Bias-Independent Components of MOSFET Series Resistance From S-Parameters", IEEE Trans. on Electron Devices, vol. 53, no. 3, pp.571-573, March 2006

\section{Acknowledgement}

This work was supported by the Engineering and Physical Science Research Council, UK. 